\title{
Analytical calculation of tube counts and geometric characteristics of tube layouts of heat exchangers
}

\author{
Andrew Ch. Yiannopoulos
}

\begin{abstract}
The optimum design of shell-and-tube heat exchangers is an interesting subject in engineering, because it is interrelated with constructional, operational and economic issues. In the present work a detailed geometric consideration of tube layouts is conducted and a method for precise calculation of tube counts is given. Furthermore, some issues concerning the design of tube-sheets and baffles are examined. The calculations were performed through a mathematical approach by introducing a characteristic parameter, the value of which depends on the number of tubes and therefore can be given in tables. A simple working equation was also derived for the calculation of shell diameter of a heat exchanger for a given number of tubes, tube diameter and tube pitch. The results, compared with those obtained by approximate relationships or tables available in the literature, showed that the method of the present study is complete, rapid and with the best accuracy.
\end{abstract}

Index Terms - Heat exchanger, Square pattern, Triangular pattern, Tube counts, Tube layout.

\section{INTRODUCTION}

The study of heat exchangers is of great interest, because the conversion of heat is widely used in industries or many other facilities. Among them chemical industries, refineries, power plants and factories for production of many kinds of products are the main places where the heat exchangers are used. In the early years the design of heat exchangers was based on empirical methods, so they had either low efficiency or many clogging and fouling problems. A systematic methodology was presented in 1950 by Kern [1], which was established as the common practice for designing heat exchangers for the next years and named "Kern's method". He gave the calculation of heat transfer coefficients, pressure drop and many other constructional features of heat exchangers, such as shell dimensions, tube patterns, tube counts etc. In 1963 the professor Bell [2], in the University of Delaware in USA, by accomplishing a project, presented a more precise method than Kern's method, which is commonly described as the "Bell-Delaware Method". Bell took into account some more conditions and parameters that had not been included in the Kern's method. Among them are the leakage effects of fluids due to by-pass or due to clearances between baffles and shell or between baffle holes and tubes etc. For tube counts Bell applied the available tables. Serna and Jiménez [3] adopted Bell's method and presented a compact formulation for the design and optimization of heat exchangers. For the calculation of tube counts they used an empirical expression suggested earlier by Taborek [4].

A. Ch. Yiannopoulos, Department of Mechanical Engineering, Technological Education Institution of Western Greece, M. Alexandrou 1, Patras 26334, Greece, Phone: +30-2610-369084, Fax: +30-2610-369198,
For large number of tubes the simple method of plotting the layout and counting the tubes is very cumbersome and time-consuming. In Wolverine Data Book III, by Thome [5], as well as in the Books by Kakac and Liu [6], Shah and Sekulic [7], Serth and Lestina [8], one can find some approximate analytical expressions or tables available for the estimation of tube counts, which often cover only certain standard combinations of pitch, tube diameter, and layout parameters. There are also many Handbooks, in which several working methods for designing heat exchangers are given. The most common of them is the Perry's Chemical Engineers Handbook [9] and the Coulson \& Richardson's Chemical Engineering, Vol. 6, by Sinnott [10]. In these Handbooks, a detailed analysis for heat exchangers is included, as well as tube patterns and tables with tube counts, and other technical characteristics. It is of interest the work of Phadke [11], who suggested a mathematical method to predict the number of tubes and presented tables with tube counts for various combinations of tube pattern parameters. His method can accommodate any configuration of tube layout. For the design of shell-and-tube heat exchangers, Muralikrishna and Shenoy [12], Serna-Gonzalez et al. [13], Sahin et al. [14], Fettaka et al. [15] calculated the number of tubes by using approximate empirical equations. The Tubular Exchanger Manufacturer's Association (TEMA) [16] has standardised the heat exchangers and published rules and technical specifications available for the scientific community. Tan and Fok [17] and Than et al. [18], in the design of heat exchangers adopted the empirical expressions of TEMA standards for tube counts, whereas Guo et al. [19] used a different empirical equation which is not accurate enough.

In the present work a detailed geometric consideration is conducted to predict either the total number of tubes (tube counts) or the number of tubes on particular rows and/or in the baffle window. To do this a precise method is developed for the calculation of geometric characteristics for several tube layouts, namely the square and triangular tube pattern. The method is based on the mathematical computation of distances of all holes from the center of a circular tube-sheet. For each tube pattern there are discrete circles which contain a certain number of holes for a given pitch size. The number of holes varies slightly if there is or not a hole at the center of the cross-section. To the best of our knowledge, the distinction and detailed consideration of two cases, with or without a hole at the center of a tube-sheet, is first examined in the present study. For the solution, a characteristic parameter $f$ was introduced, which depends only on the number of holes and tube pattern. This proved to be a great advantage because parameter $f$ can be tabulated and used directly to determine the bundle or shell diameter, if the number of tubes has been formerly defined through thermal consideration of the heat exchanger. 


\section{METHOD OF ANALYSIS}

\section{A. Tube layouts}

The following analysis deals with the shell-and-tube heat exchangers, which have a number of tubes fitted onto circular tube-sheets at the two ends. The tube-sheets have an equal number of holes to accommodate the tubes. The most common tube layouts are the square and triangular patterns, which are divided further into two forms. The one has a hole at the center of the cross-section, whereas the other not. These configurations are examined separately, because the method of analysis and the results differ slightly. To distinguish them we introduce the abbreviations:

SQ-1 $=1^{\text {st }}$ kind of square pattern with a hole at the center.

SQ-2 $=2^{\text {nd }}$ kind of square pattern without a hole at the center. The intersection of axes is placed at the center of the square.

TR-1 $=1^{\text {st }}$ kind of triangular pattern with a hole at the center. $\mathrm{TR}-2=2^{\text {nd }}$ kind of triangular pattern without a hole at the center. The intersection of axes is placed at the center of the equilateral triangle.

In the square patterns SQ-1 and SQ-2 the tube rows are parallel to the orthogonal axes $(x, y)$ and they have the same tube pitch $t$ along $x$ and $y$ directions, as in Figs. 1(a) and 1(b). The triangular patterns TR-1 and TR-2 are formed by equilateral triangles with rows parallel to $\left(x^{\prime}, y^{\prime}\right)$ axes, which are inclined by $60^{\circ}$ with respect to $x$ axis, and they have the same tube pitch $t$ along $x, x^{\prime}$ and $y^{\prime}$ directions, as in Figs. 2(a) and 2(b).

\section{B. Number of holes included in a circle}

For a given shell diameter there is a limit to the number of tubes that can fit, which depends on heat exchanger design parameters, as the tube diameter, pitch, layout and clearances. The objective of this section is to find how many holes of diameter $d_{0}$ can be included into a circle of diameter $D$. Obviously the number of holes depends on pitch $t$ and therefore the diameter $D$ is related with the size of $t$. This relation can be expressed through a characteristic parameter $f$ which depends on the number of holes that can be accommodated on a tube-sheet. To calculate the parameter $f$ two positive integers $A$ and $B$ are defined, which characterise the coordinates on $(x, y)$ axes for square patterns and on $\left(x^{\prime}\right.$, $\left.y^{\prime}\right)$ axes for triangular patterns, as shown in the cross sections of Fig. 3. It is easier to work on a basic section of the circle (shaded area), where the coordinates are positive, and then to extend the calculation and obtain the total number of holes included in the circle. Taking into account the geometry of each separate pattern, the equations for the coordinates are:

$$
\begin{array}{lll}
\text { - For SQ-1: } & x=A t & y=B t \\
\text { - For SQ-2: } & x=\frac{2 A-1}{2} t & y=\frac{2 B-1}{2} t \\
\text { - For TR-1: } & x^{\prime}=A t & y^{\prime}=B t \\
\text { - For TR-2: } & x^{\prime}=\frac{3 A-1}{3} t & y^{\prime}=\frac{3 B-2}{3} t
\end{array}
$$

(a)

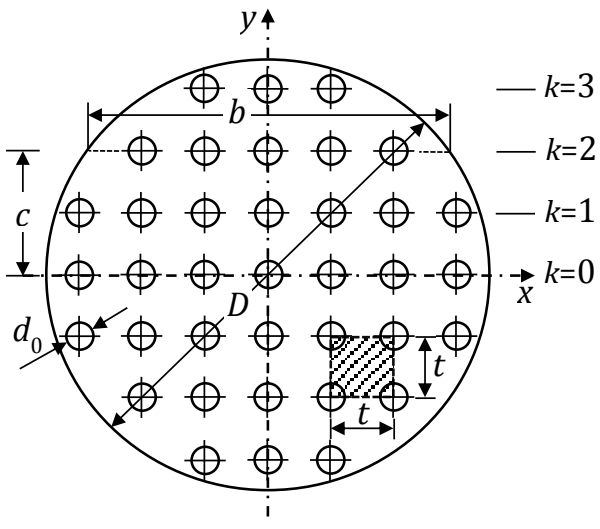

(b)

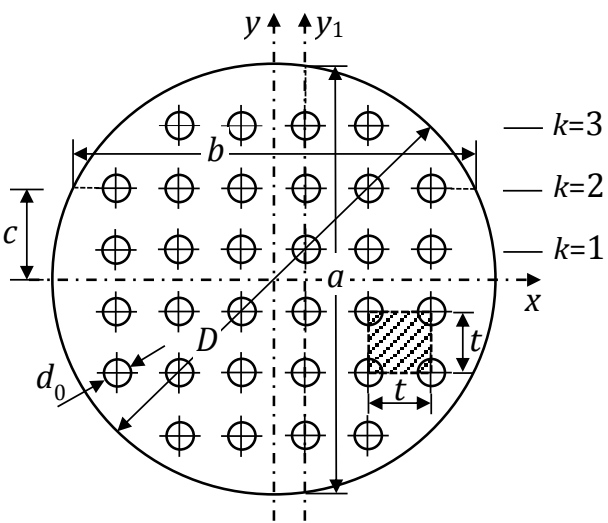

Fig. 1: Square pattern, (a) with a hole at the center (SQ-1), (b) without a hole at the center (SQ-2)

(a)

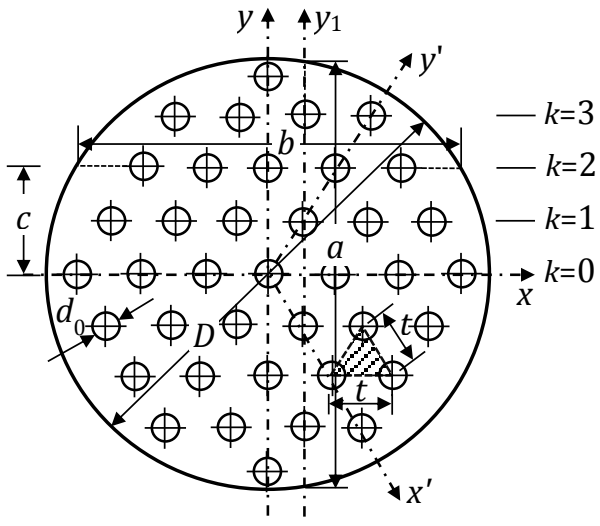

(b)

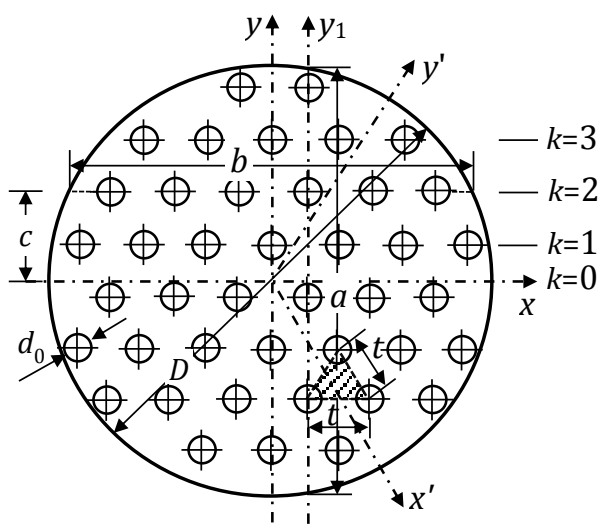

Fig. 2: Triangular pattern, (a) with a hole at the center (TR-1), (b) without a hole at the center (TR-2) 
(a)

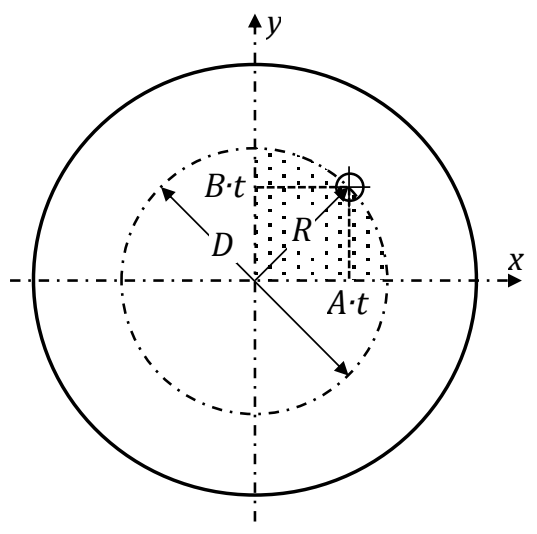

(b)

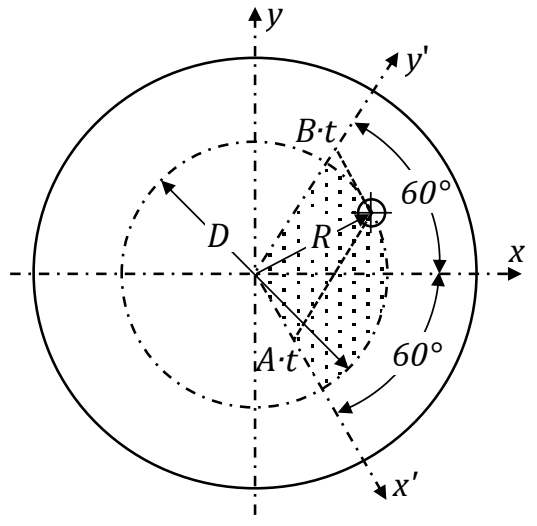

Fig. 3: Axes and coordinates, (a) for square pattern SQ-1, (b) for triangular pattern TR-1

For the examined patterns the characteristic number $f$ is expressed with the following relationships:

- For SQ-1: $\quad f=2 \sqrt{A^{2}+B^{2}}$

$$
A=0,1,2,3, \ldots, \text { and } B=0,1,2,3, \ldots
$$

- For SQ-2: $\quad f=\sqrt{(2 A-1)^{2}+(2 B-1)^{2}}$

$$
A=1,2,3, \ldots, \text { and } B=1,2,3, \ldots
$$

- For TR-1: $\quad f=2 \sqrt{A^{2}+B^{2}-A B}$

$$
A=0,1,2,3, \ldots, \text { and } B=0,1,2,3, \ldots
$$

- For TR-2:

$$
\begin{gathered}
f=\frac{2}{3} \sqrt{(3 A-1)^{2}+(3 B-2)^{2}-(3 A-1)(3 B-2)} \\
A=1,2,3, \ldots, \text { and } B=1,2,3, \ldots
\end{gathered}
$$

After the above definitions and derivations we can create a simple equation, which is valid for all patterns, and can be written as:

$$
D=f t
$$

Using equations (5) to (8) we can calculate the value $f$ for each shaded area of Fig. 3, which corresponds to a certain number of holes into this area. Then taking into account that the circle includes 4 times more holes for the square patterns SQ-1 and SQ-2, and 3 times more holes for the triangular patterns TR-1 and TR-2, we can find the total number of holes included in the circle $D$. To find the relation between $f$ and number $n$ of holes in $D$ we proceed as follows:

Step 1: We write a matrix containing the values of integers $A$ and $B$, and the values $f$ are calculated using (5) to (8). As an example the calculation for pattern SQ-1 is presented in Table I. The values of $f$ are computed for all combinations of integers $A$ and $B$, but in the table are shown only the values for $A \geq B$ due to symmetry. The table is extended to large values of $A$ and $B$, whereas a small part of it is shown here.

Table I. Values $f$ for pattern SQ-1

\begin{tabular}{cccccccc}
\hline$A$ & 0 & 1 & 2 & 3 & 4 & 5 & $\cdots$ \\
$B$ & & & & & & & \\
\hline 0 & 0 & $2 \sqrt{1}$ & $2 \sqrt{4}$ & $2 \sqrt{9}$ & $2 \sqrt{16}$ & $2 \sqrt{25}$ & \\
1 & & $2 \sqrt{2}$ & $2 \sqrt{5}$ & $2 \sqrt{10}$ & $2 \sqrt{17}$ & $2 \sqrt{26}$ & \\
2 & & & $2 \sqrt{8}$ & $2 \sqrt{13}$ & $2 \sqrt{20}$ & $2 \sqrt{29}$ & \\
3 & & & & $2 \sqrt{18}$ & $2 \sqrt{25}$ & $2 \sqrt{34}$ & \\
4 & & & & & $2 \sqrt{32}$ & $2 \sqrt{41}$ & \\
5 & & & & & & $2 \sqrt{50}$ & \\
$\vdots$ & & & & & & & \\
\hline
\end{tabular}

Step 2: We find the number $i$ of holes at the circumference of the complete circle $D$, which corresponds to any combination of integers $A$ and $B$ in the matrix. We take into account that for the center corresponds 1 hole, for $x, y$ axes and diagonal axes correspond 4 holes, whereas for the rest combinations correspond 8 holes.

Step 3: We sort the values $f$ by increasing order together with the corresponding values $i$ and then we find the values of cumulative $i$, say $n$, which are the sum of holes that included in the particular circle $D$. The results are shown in Table II.

Table II. Values $f$ sorted by increasing order together with $i$ and number of holes $n$ for pattern SQ-1

\begin{tabular}{ccc}
\hline$f$ & $i$ & $n$ \\
0 & 1 & 1 \\
$2 \sqrt{1}$ & 4 & 5 \\
$2 \sqrt{2}$ & 4 & 9 \\
$2 \sqrt{4}$ & 4 & 13 \\
$2 \sqrt{5}$ & 8 & 21 \\
$2 \sqrt{8}$ & 4 & 25 \\
$2 \sqrt{9}$ & 4 & 29 \\
$2 \sqrt{10}$ & 8 & 37 \\
$2 \sqrt{13}$ & 8 & 45 \\
$2 \sqrt{16}$ & 4 & 49 \\
$2 \sqrt{17}$ & 8 & 57 \\
$2 \sqrt{18}$ & 4 & 61 \\
$\vdots$ & $\vdots$ & $\vdots$ \\
\hline
\end{tabular}

Proceeding with the same manner we can find the number $n$ of the holes for the patterns SQ-2, TR-1 and TR-2. It is clear that each $n$ corresponds to a certain $f$, which is different for each particular pattern. The computations were carried out by a computer program for an increasing large number $n$ and the results have been tabulated in the Appendix. 


\section{Shell inside diameter}

The design of heat exchangers must include the formation of tube-sheets. Therefore, if the number $n$ of tubes is given, using (9) we can obtain directly the diameter $D$ which refers to the center line of the outer tubes of the bundle. However, the formation of tube-sheets needs some more details to account for, as for instance, the diametric clearance $C$ between tube bundle and shell, the clearance along a diameter to fit a separation plate of flow etc. Such considerations are important because they influence the magnitude of the inside diameter of the shell.

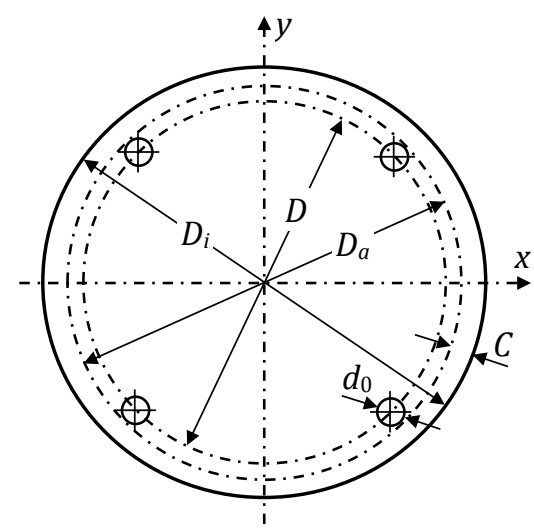

Fig. 4: Geometry of a tube-sheet

Fig. 4 shows the geometry of a tube-sheet fitted into the shell of a heat exchanger. If we add in (9) the outer diameter $d_{0}$ of the tubes and the proper clearance $C$, we obtain the complete working equation for the heat exchanger, as:

$$
D_{i}=f t+d_{0}+2 C
$$

where $D_{i}$ is the shell inside diameter. The tube bundle diameter $D_{b}$ is given by (10) for $C=0$. It can be noticed that using the appended tables with the characteristic parameter $f$, the calculations become easier and more precise than other approximate solutions.

\section{Number of rows of a tube-sheet}

For the study of heat exchangers it is important to know how many rows of tubes should be placed on a tube-sheet and how many holes should be drilled on each row. In this section we calculate the number of rows parallel to $x$ axis for each particular pattern. In the square patterns SQ-1 and SQ-2 the horizontal rows are identical to the vertical rows, due to symmetry with respect to $x$ and $y$ axis. In the triangular patterns TR-1 and TR-2 the horizontal rows are not identical to the inclined rows parallel to $x^{\prime}$ and $y^{\prime}$ axis, so there is some difference in the procedure.

Square pattern $S Q-1$. Fig. 1(a) shows the geometry of SQ-1 pattern. To find the number of tube rows we work on $y$ axis and above $x$ axis. We divide the radius $R$ by the pitch $t$ and we obtain a number from which we take the integer part. Then we replace $R$ by $D / 2$ and using (9) we can write the equation for the number of rows above $x$ axis, say $m_{1}$, as:

$$
m_{1}=\operatorname{Int}\left[\frac{f}{2}\right]
$$

The total number of rows that can be fitted on a tube-sheet is then calculated by duplicating $m_{1}$ and adding one row for that on $x$ axis. So we obtain the total number $m$ of tube rows parallel to $x$ axis on a tube-sheet as:

$$
m=1+2 \operatorname{Int}\left[\frac{f}{2}\right]
$$

Square pattern SQ-2. Fig. 1(b) shows the geometry of SQ-2 pattern. To find the number of tube rows for this pattern we work similarly above $x$ axis but on $y_{1}$ axis. Since there is no row on $(x, y)$ axes we divide $a / 2+t / 2$ by pitch $t$ and we take the integer part from the result. The distance $a$ is the length of the vertical chord on $y_{1}$ axis and is given by the equation:

$$
a=2 \sqrt{R^{2}-\left(\frac{t}{2}\right)^{2}}
$$

We replace $R$ by $D / 2$ and using (9) we have the number of rows above $x$ axis, say $m_{1}$, as:

$$
m_{1}=\operatorname{Int}\left[\frac{1+\sqrt{f^{2}-1}}{2}\right]
$$

The total number $m$ of rows of the tube-sheet is then obtained by duplicating the number $m_{1}$, as:

$$
m=2 \operatorname{Int}\left[\frac{1+\sqrt{f^{2}-1}}{2}\right]
$$

Triangular pattern TR-1. The number of tube rows for this pattern is determined with the same manner. We work separately on $y$ and $y_{1}$ axis and above $x$ axis. As Fig. 2(a) shows, the distance between rows parallel to $x$ axis is equal to the height $h$ of the equilateral triangle, which is: $h=t \sqrt{3} / 2$.

(i) On y axis. We divide $R$ by $2 h$ and we find the number of rows, say $m_{1(\mathrm{y})}$, as:

$$
m_{1(y)}=\operatorname{Int}\left[\frac{\sqrt{3} f}{6}\right]
$$

(ii) On $y_{l}$ axis. We use (13) and divide $a / 2+h$ by $2 h$ and the number of rows, say $m_{1(\mathrm{y} 1)}$, is:

$$
m_{1\left(y_{1}\right)}=\operatorname{Int}\left[\frac{3+\sqrt{3} \sqrt{f^{2}-1}}{6}\right]
$$

Therefore, the number of rows above $x$ axis is the sum of the two numbers $m_{1(\mathrm{y})}$ and $m_{1(\mathrm{y} 1)}$, so we have the equation:

$$
m_{1}=\operatorname{Int}\left[\frac{\sqrt{3} f}{6}\right]+\operatorname{Int}\left[\frac{3+\sqrt{3} \sqrt{f^{2}-1}}{6}\right]
$$

The total number $m$ of rows parallel to $x$ axis of the tube-sheet is then obtained by duplicating the number $m_{1}$ and adding one unit for the row on $x$ axis, as:

$$
m=1+2\left\{\operatorname{Int}\left[\frac{\sqrt{3} f}{6}\right]+\operatorname{Int}\left[\frac{3+\sqrt{3} \sqrt{f^{2}-1}}{6}\right]\right\}
$$

Triangular pattern TR-2. In this case the $x$ axis passes through the center of the equilateral triangle, as Fig. 2(b) shows, and since there is no symmetry about $x$ axis, we work on $y$ and $y_{1}$ axis separately for calculations above or below $x$ axis. The distance between rows parallel to $x$ axis is also equal to the height $h$ of the equilateral triangle. Then we proceed according to the previous manner: 


\section{(i) Above $x$ axis}

We examine the horizontal rows of the holes on $y$ axis and divide $R+h+h / 3$ by $2 h$, so we find the number of them, as:

$$
m_{1(y)}=\operatorname{Int}\left[\frac{4+\sqrt{3} f}{6}\right]
$$

After that we examine the horizontal rows on $y_{l}$ axis. We use (13) and divide $a / 2+h / 3$ by $2 h$, so we find the number of them, as:

$$
m_{1\left(y_{1}\right)}=\operatorname{Int}\left[\frac{1+\sqrt{3} \sqrt{f^{2}-1}}{6}\right]
$$

We add (20) and (21) and obtain the number $m_{1}$ of all rows above $x$ axis, which is:

$$
m_{1}=\operatorname{Int}\left[\frac{4+\sqrt{3} f}{6}\right]+\operatorname{Int}\left[\frac{1+\sqrt{3} \sqrt{f^{2}-1}}{6}\right]
$$

(ii) Below $x$ axis

We examine the horizontal rows on $y$ axis and divide $R+2 h / 3$ by $2 h$, so we find the number of rows, as:

$$
m_{2(y)}=\operatorname{Int}\left[\frac{2+\sqrt{3} f}{6}\right]
$$

We continue with the examination of the horizontal rows on $y_{1}$ axis and using (13) we divide $a / 2+h+2 h / 3$ by $2 h$. The number of rows is:

$$
m_{2\left(y_{1}\right)}=\operatorname{Int}\left[\frac{5+\sqrt{3} \sqrt{f^{2}-1}}{6}\right]
$$

We add (23) and (24) and obtain the number $m_{2}$ of all rows below $x$ axis, as:

$$
m_{2}=\operatorname{Int}\left[\frac{2+\sqrt{3} f}{6}\right]+\operatorname{Int}\left[\frac{5+\sqrt{3} \sqrt{f^{2}-1}}{6}\right]
$$

The total number $m$ of rows parallel to $x$ axis of the tube-sheet is then obtained as the sum of the two numbers $m_{1}$ and $m_{2}$ :

$$
m=m_{1}+m_{2}
$$

It is worth noting that number $m$ of all patterns depends only on parameter $f$.

\section{E. Number of holes on any row}

The number of holes that can be fitted on any row of the tube-sheet is also calculated separately for each pattern. For this case we examine the rows parallel to $x$ axis, and define $c$ as the distance of a particular row from $x$ axis and $b$ as the length of the chord at $c$, as shown in Figs. 1 and 2.

Square pattern $S Q-1$. We work on rows above $x$ axis. The rows are numbered by $k$, so the central row on $x$ axis takes the number $k=0$ and the last takes the number $k=m_{1}$. Considering the geometry of Fig. 1(a) and replacing $R=D / 2$ in (9), we write the following relationships:

$$
b=2 \sqrt{R^{2}-c^{2}} \quad R=\frac{1}{2} f t \quad c=k t
$$

We take into account (27) and divide the half width $b / 2$ of the chord by $t$ to obtain a number from which we take the integer part. Then we duplicate this integer and add one unit for the hole on $y$ axis, so we have the number $z_{k}$ of the holes on a particular row $k$, as:

$$
z_{k}=1+2 \operatorname{Int}\left[\sqrt{\left(\frac{f}{2}\right)^{2}-k^{2}}\right]
$$

where $k=0,1,2,3, \ldots, m_{1}$, and $m_{1}$ is given by (11).

The number of holes on the central row, namely on $x$ axis, is obtained from (28) for $k=0$, as:

$$
z_{0}=1+2 \operatorname{Int}\left[\frac{f}{2}\right]
$$

The arrangement SQ-1 is symmetrical about $x$ and $y$ axis, so the number $m$ of rows is equal to the number $z_{0}$ of holes at the central row, i.e. $z_{0}=m$, and thus (29) is identical to (12).

Square pattern $\mathbf{S} \boldsymbol{Q}-\mathbf{2}$. We work as before on rows above $x$ axis. The rows are numbered by $k$, the first row takes $k=1$ and the last $k=m_{1}$. From the geometry of Fig. 1(b) we have the relationships:

$$
b=2 \sqrt{R^{2}-c^{2}} \quad R=\frac{1}{2} f t \quad c=\frac{2 k-1}{2} t
$$

We use (30) and divide $b / 2+t / 2$ by $t$, and then we take the integer part from the result. We duplicate the integer and the number $z_{k}$ of the holes on any row $k$ is:

$$
z_{k}=2 \operatorname{Int}\left[\frac{1+\sqrt{f^{2}-(2 k-1)^{2}}}{2}\right]
$$

where $k=1,2,3, \ldots, m_{1}$, and $m_{1}$ is given by (14).

The number of holes on the first row, near $x$ axis, is obtained from (31) for $k=1$, as:

$$
z_{1}=2 \operatorname{Int}\left[\frac{1+\sqrt{f^{2}-1}}{2}\right]
$$

We note that the number $m$ of rows is equal to the number $z_{1}$ of holes of the first row, since the layout SQ-2 is symmetrical about $x$ and $y$ axes, which means $z_{1}=m$, and therefore (32) is identical to (15).

Triangular pattern TR-1. We work as above on rows above $x$ axis. The rows are numbered by $k$, the central row takes $k=0$ and the last $k=m_{1}$. From the geometry of Fig. 2(a) we have the relationships:

$$
b=2 \sqrt{R^{2}-c^{2}} \quad R=\frac{1}{2} f t \quad c=\frac{\sqrt{3}}{2} k t
$$

We observe that for $k=0,2,4, \ldots$, we have holes on $y$ axis and for $k=1,3,5, \ldots$, we have holes on $y_{1}$ axis. So we work separately if $k$ is an odd or even number.

- For $k=1,3,5, \ldots$, taking into account (33) and dividing $b / 2+t / 2$ by $t$, we obtain a number from which we take the integer part. We duplicate this integer and write the number $z_{k}$ of the holes on a particular row $k$, as:

$$
z_{k}=2 \operatorname{Int}\left[\frac{1+\sqrt{f^{2}-3 k^{2}}}{2}\right], \quad k=1,3,5, \ldots
$$

- For $k=0,2,4, \ldots$, and following the same way we divide $b / 2$ by $t$ to obtain a number and the integer part of it. We duplicate it and add one unit for the hole on $y$ axis. The 
number $z_{k}$ of the holes on a particular row $k$ is:

$$
z_{k}=1+2 \operatorname{Int}\left[\frac{\sqrt{f^{2}-3 k^{2}}}{2}\right], \quad k=0,2,4, \ldots
$$

Triangular pattern TR-2. The analysis of this pattern differs if the rows are above or below $x$ axis. So we work separately for these two cases. The rows are numbered by $k$, the first row takes $k=1$ and the last $k=m_{1}$. From the geometry of Fig. 2(b) we can write the same relationships as in TR-1 for $b$ and $R$, but there is a difference in $c$.

- Above x axis: $\quad c=\frac{(3 k-1) \sqrt{3}}{6} t$

- Below x axis: $\quad c=\frac{(3 k-2) \sqrt{3}}{6} t$

\section{(i) Above $x$ axis}

We observe that for $k=1,3,5, \ldots$, we have holes on $y$ axis and for $k=2,4,6, \ldots$, we have holes on $y_{1}$ axis. So we work separately if $k$ is an odd or even number.

- For $k=1,3,5, \ldots$, we use (36) and divide $b / 2$ by $t$. Then we take the integer part from the result. We duplicate the integer and add one unit for the central hole. The number $z_{k}$ of the holes on a particular row $k$ can be written as:

$$
z_{k}=1+2 \operatorname{Int}\left[\frac{\sqrt{f^{2}-\frac{1}{3}(3 k-1)^{2}}}{2}\right], \quad k=1,3,5, \ldots
$$

- For $k=2,4,6, \ldots$, we use (36) and divide $b / 2+t / 2$ by $t$ to obtain a number and the integer part of it. We duplicate the integer and then we have $z_{k}$, as:

$$
z_{k}=2 \operatorname{Int}\left[\frac{1+\sqrt{f^{2}-\frac{1}{3}(3 k-1)^{2}}}{2}\right], \quad k=2,4,6, \ldots
$$

\section{(ii) Below $x$ axis}

We observe that we have holes on $y_{1}$ axis for $k=1,3,5, \ldots$, and on $y$ axis for $k=2,4,6, \ldots$, and therefore we must work separately if $k$ is an odd or even number.

- For $k=1,3,5, \ldots$, we use (36) and divide $b / 2+t / 2$ by $t$. After that we take the integer part from the result. We duplicate it and the number $z_{k}$ of the holes on a particular row $k$ is:

$$
z_{k}=2 \operatorname{Int}\left[\frac{1+\sqrt{f^{2}-\frac{1}{3}(3 k-2)^{2}}}{2}\right], \quad k=1,3,5, \ldots
$$

- For $k=2,4,6, \ldots$, we use (36) and divide $b / 2$ by $t$, and then we take the integer part from the result. We duplicate it, and add one unit for the central hole, so the number $z_{k}$ is:

$$
z_{k}=1+2 \operatorname{Int}\left[\frac{\sqrt{f^{2}-\frac{1}{3}(3 k-2)^{2}}}{2}\right], \quad k=2,4,6, \ldots
$$

\section{F. Number of holes in a circular segment}

The number of holes which are included in a segment of a circle can be calculated according to the previous analysis.
We usually need to know this number if we are looking for the number of tubes passing through a baffle window of a heat exchanger. If $B_{c}$ is the height of the baffle cut, the remaining distance above $x$ axis is $R-B_{c}$. The geometry of a segmental baffle is shown in Fig. 5, where the shaded area is the cut. The concept is to find how many horizontal rows are fitted along the distance $R-B_{c}$ first and then to find the number of rows in the baffle segment by subtraction from the total number $m_{1}$ of rows above $x$ axis. Since this number is already determined, we work separately for each pattern, as:

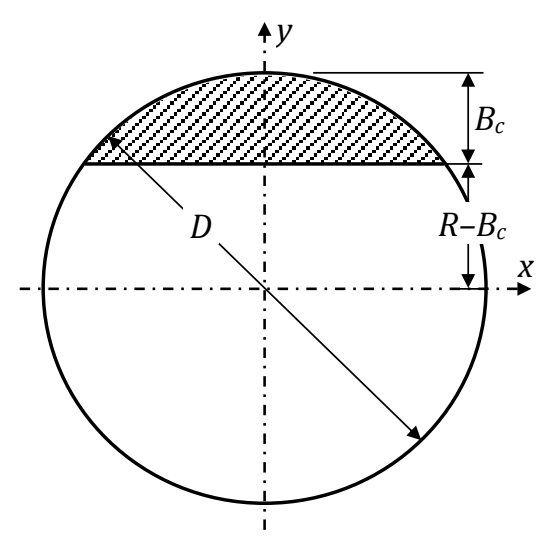

Fig. 5: Segmental baffle geometry

Square pattern $S Q-1$. We divide the distance $R-B_{c}$ by $t$ and take the integer part from the result to obtain the number $j$ of rows parallel to $x$ axis that can be fitted along the distance $R-B_{c}$. For $j$ the following equation can be written:

$$
j=\operatorname{Int}\left[\frac{D-2 B_{C}}{2 t}\right]
$$

The total number of rows above $x$ axis is known by (11) as $m_{1}$ and consequently the rows from $j+1$ up to $m_{1}$ are included in the baffle window. Each of them has a particular number of holes given by (28). The sum gives the number of holes included in the circular segment, say $N_{c}$, as:

$$
\begin{gathered}
N_{c}=\sum_{k}^{m_{1}}\left[1+2 \operatorname{Int}\left[\frac{\sqrt{f^{2}-4 k^{2}}}{2}\right]\right] \\
\text { where } k=j+1, j+2, j+3, \ldots, m_{1}
\end{gathered}
$$

If the baffle cut is in the opposite, i.e. below $x$ axis, the equation (42) for $N_{c}$ remains the same, due to symmetry of the arrangement of holes with respect to $x$ axis.

Square pattern $S Q-2$. For this case we divide $R-B_{c}+t / 2$ by $t$ and after taking the integer part we find the number $j$ of rows parallel to $x$ axis, and along the distance $R-B_{c}$, as:

$$
j=\operatorname{Int}\left[\frac{D-2 B_{c}+t}{2 t}\right]
$$

The total number of rows above $x$ axis is already known by (14) as $m_{1}$. Then the rows from $j+1$ up to $m_{1}$ are included in the baffle window and each of them has a particular number of holes given by (31). The sum gives the number of holes included in the circular segment, say $N_{c}$, as: 


$$
N_{c}=\sum_{k}^{m_{1}} 2 \operatorname{Int}\left[\frac{1+\sqrt{f^{2}-(2 k-1)^{2}}}{2}\right]
$$

where $k=j+1, j+2, j+3, \ldots, m_{1}$

If the baffle cut is in the opposite, i.e. below $x$ axis, the equation (44) for $N_{c}$ is also valid, due to symmetry of the arrangement of holes with respect to $x$ axis.

Triangular pattern TR-1. Following the same procedure as before, we divide for this pattern the distance $R-B_{c}$ by the height of the equilateral triangle $h=t \sqrt{3} / 2$ and take the integer part. The number $j$ of rows parallel to $x$ axis is:

$$
j=\operatorname{Int}\left[\frac{\sqrt{3}\left(D-2 B_{C}\right)}{3 t}\right]
$$

The total number of rows above $x$ axis is already determined by (18) as $m_{1}$, and consequently the rows from $j+1$ up to $m_{1}$ are included in the baffle window. Each of them has a particular number of holes given by (34) and (35). The sum gives the number of holes included in the circular segment, say $N_{c}$, as:

$$
\begin{gathered}
N_{c}=\left\{\begin{array}{cc}
\sum_{k}^{m_{1}}\left[1+2 \operatorname{Int}\left[\frac{\sqrt{f^{2}-3 k^{2}}}{2}\right]\right] & \text { for } k=1,3,5, \ldots \\
+\sum_{k}^{m_{1}} 2 \operatorname{Int}\left[\frac{1+\sqrt{f^{2}-3 k^{2}}}{2}\right] & \text { for } k=2,4,6, \ldots
\end{array}\right. \\
\text { where } k=j+1, j+2, j+3, \ldots, m_{1}
\end{gathered}
$$

If the baffle cut is in the opposite, below $x$ axis, the number $N_{c}$ is also given by (46), due to symmetry of the hole layout with respect to $x$ axis.

Triangular pattern TR-2. For the triangular pattern TR-2 there is a different number of holes in the circular segment if it is cut above or below $x$ axis. For this reason we proceed separately for these two cases.

(i) Above $x$ axis

The height of the equilateral triangle is $h=t \sqrt{3} / 2$. We divide the distance $R-B_{c}+h / 3$ by $h$, take the integer part, and the number $j$ of rows parallel to $x$ axis can be written as:

$$
j=\operatorname{Int}\left[\frac{\sqrt{3}\left(D-2 B_{C}\right)+t}{3 t}\right]
$$

The total number of rows above $x$ axis is given by (22) as $m_{1}$, and thus the rows from $j+1$ up to $m_{1}$ are included in the circular segment. Each of them has a particular number of holes given by (37) and (38) and therefore the sum gives the number of holes within the circular segment, say $N_{c}$, as:

$N_{c}= \begin{cases}\sum_{k}^{m_{1}}\left[1+2 \operatorname{Int}\left[\frac{\sqrt{f^{2}-\frac{1}{3}(3 k-1)^{2}}}{2}\right]\right] & \text { for } k=1,3,5, \ldots \\ +\sum_{k}^{m_{1}} 2 \operatorname{Int}\left[\frac{1+\sqrt{f^{2}-\frac{1}{3}(3 k-1)^{2}}}{2}\right] & \text { for } k=2,4,6, \ldots\end{cases}$

$$
\text { where } k=j+1, j+2, j+3, \ldots, m_{1}
$$

\section{(ii) Below $x$ axis}

For this case we divide the distance $R-B_{c}+2 h / 3$ by $h$ and taking the integer part we have the number $j$ of rows parallel to $x$ axis, as:

$$
j=\operatorname{Int}\left[\frac{\sqrt{3}\left(D-2 B_{C}\right)+2 t}{3 t}\right]
$$

The total number of rows below $x$ axis is given by (25) as $m_{2}$. As before, the rows from $j+1$ up to $m_{2}$ are included in the baffle window and each of them has a particular number of holes given by (39) and (40). Consequently, the sum gives the holes included in the circular segment, say $N_{c}$, as:

$$
N_{c}=\left\{\begin{array}{cc}
\sum_{k}^{m_{2}} 2 \operatorname{Int}\left[\frac{1+\sqrt{f^{2}-\frac{1}{3}(3 k-2)^{2}}}{2}\right] & \text { for } k=1,3,5, \ldots \\
+\sum_{k}^{m_{2}}\left[1+2 \operatorname{Int}\left[\frac{\sqrt{f^{2}-\frac{1}{3}(3 k-2)^{2}}}{2}\right]\right] & \text { for } k=2,4,6, \ldots \\
\text { where } k=j+1, j+2, j+3, \ldots, m_{2}
\end{array}\right.
$$

\section{NUMERICAL EXAMPLES AND DISCUSSION}

Example 1. A heat exchanger should have $n=347$ tubes in order to satisfy the heat transfer requirements. The tubes are made from seamless steel and according to ISO standards they have an outer diameter $d_{0}=21.3 \mathrm{~mm}$ and thickness $s=2$ $\mathrm{mm}$. The clearance between tubes is taken $\delta=10 \mathrm{~mm}$ and the allowed diametric clearance between tube bundle and shell must be $C=15 \mathrm{~mm}$. The height of the baffle window is $25 \%$ of the diameter. The tube pitch is: $t=d_{0}+\delta=21.3+10=31.3 \mathrm{~mm}$.

By using the previous analysis we calculate for SQ-1 and TR-1 the layout characteristics, namely: Shell inside diameter $D_{i}$, diameter $D_{b}$ of the tube bundle, total number $m$ of rows of the tube-sheet, number $z_{0}$ of tubes on the central row, number $N_{\mathrm{c}}$ of tubes in the baffle window.

\section{(a) Square pattern $S Q-1$ :}

From Table A.1: $f=20.88061$ for $n=349$, which is just greater than given $n$.

From $(10): \quad D_{i}=20.88061 \times 31.3+21.3+2 \times 15=$

$$
=704.86 \mathrm{~mm}
$$

From (10): $\quad D_{b}=20.88061 \times 31.3+21.3=674.86 \mathrm{~mm}$

From (12): $\quad m=1+2 \times \operatorname{Int}\left[\frac{20.88061}{2}\right]=21$

From (29): $\quad z_{0}=m=21$

The height of the baffle window is:

$$
B_{c}=25 \% D_{b}=0.25 \times 674.86=168.72 \mathrm{~mm}
$$

From (41):

$$
j=\operatorname{Int}\left[\frac{674.86-2 \times 168.72}{2 \times 31.3}\right]=5
$$

From (11): $\quad m_{1}=\operatorname{Int}\left[\frac{20.88061}{2}\right]=10$

Thus the calculation of $N_{c}$ is performed for $k=6,7,8,9,10$.

From (42): $N_{c}=\sum_{k=6}^{10}\left[1+2 \times \operatorname{Int}\left[\frac{\sqrt{20.88061^{2}-4 k^{2}}}{2}\right]\right] \Rightarrow$

$$
\Rightarrow N_{c}=17+15+13+11+7=63
$$

\section{(b) Triangular pattern TR-1:}

From Table A.3: $f=19.28730$ for $n=349$, which is just greater than given $n$. 
From (10): $\quad D_{i}=19.28730 \times 31.3+21.3+2 \times 15=$ $=654.99 \mathrm{~mm}$

From (10): $\quad D_{b}=19.28730 \times 31.3+21.3=624.99 \mathrm{~mm}$

From (18):

$$
m_{1}=\operatorname{Int}\left[\frac{\sqrt{3} \times 19.28730}{6}\right]+\operatorname{Int}\left[\frac{3+\sqrt{3} \sqrt{19.28730^{2}-1}}{6}\right]=11
$$

From (19): $\quad m=1+2 m_{1}=1+2 \times 11=23$

From (35) and for $k=0: z_{0}=1+2 \times \operatorname{Int}\left[\frac{19.28730}{2}\right]=19$

The height of the baffle window is:

$$
B_{c}=25 \% D_{b}=0.25 \times 624.99=156.25 \mathrm{~mm}
$$

From (45): $\quad j=\operatorname{Int}\left[\frac{\sqrt{3}(624.99-2 \times 156.25)}{3 \times 31.3}\right]=5$

Thus the calculation of $N_{c}$ must be performed for $k=j+1$ to $m_{1}$, which means $k=6,7,8, \ldots, 11$.

From (46):

$N_{c}=\left\{\begin{array}{cc}\sum_{k=7}^{11}\left[1+2 \operatorname{Int}\left[\frac{\sqrt{19.28730^{2}-3 k^{2}}}{2}\right]\right] & \text { for } k=1,3,5, \ldots \\ +\sum_{k=6}^{11} 2 \operatorname{Int}\left[\frac{1+\sqrt{19.28730^{2}-3 k^{2}}}{2}\right] & \text { for } k=2,4,6, \ldots\end{array}\right.$

which gives the result:

$$
N_{c}=15+11+3+2 \cdot(8+7+4)=67
$$

We observe that the arrangement according to TR-1 pattern gives the smallest shell inside diameter and can accommodate two more tubes than 347, namely 349 tubes.

Example 2. To compare our results with the values taken from available tables of tube counts or with the results extracted from approximate empirical equations, we consider a heat exchanger with adapted parameters, as: Shell inside diameter $D_{i}=13.25^{\prime \prime}=336.55 \mathrm{~mm}$, triangular pattern TR-1, tubes of outer diameter $d_{0}=3 / 4 "=19.05 \mathrm{~mm}$, pitch $t=1 "=25.4$ $\mathrm{mm}$ and clearance $C=0$. From (10) we have: $D_{b}=D_{i}$.

The characteristic parameter $f$ which corresponds to the data of the heat exchanger can be calculated by applying (10) for $C=0$, as:

$$
f=\frac{D_{i}-d_{0}}{t}=\frac{336.55-19.05}{25.4}=12.5
$$

From the appended Table A. 3 and for $f=12.49<12.5$ our method gives $n=151$ tubes.

(a) Using the tables of tube counts by Serth and Lestina [8], and for the same data, we find $n=136$. There is a deviation of about $10 \%$ or 15 tubes less.

(b) The application of an empirical equation cited in the Handbook by Sinnott [10], for bundle diameter $D_{b}=336.55$ $\mathrm{mm}, d_{0}=19.05 \mathrm{~mm}$, and the given factors $K_{1}=0.319$ and $n_{1}=2.142$ for the triangular pattern with a pitch $t=1.25 d_{0}$, gives the following result:

$$
n=K_{1}\left(\frac{D_{b}}{d_{0}}\right)^{n_{1}}=0.319\left(\frac{336.55}{19.05}\right)^{2,142}=149.69 \approx 150
$$

To compare this result with our method the pitch $t$ must be adapted, because in this case it is slightly different from the aforementioned value, which is: $t=1.25 \times 19.05=23.81 \mathrm{~mm}$. From (10) we find the new parameter $f$ :

$$
f=\frac{D_{i}-d_{0}}{t}=\frac{336.55-19.05}{23.81}=13.33
$$

From Table A.3 and for $f=13.11488<13.33$ our method results to $n=163$ tubes. We observe that the empirical equation gave 13 tubes less, which corresponds to a deviation of about $8 \%$.

(c) The comparison with the approximate equation in Perry's Handbook [9], for the triangular pattern with a pitch $t=1.25 d_{0}$, requires the value of a parameter $C_{0}$ :

$$
C_{0}=0.75 \frac{D_{b}}{d_{0}}-36=0.75 \frac{336.55}{19.05}-36=-22.75
$$

The range of accuracy is $-24 \leq C_{0} \leq 24$, which is valid. The number of tubes is given by the following relationship:

$$
n=1298+74.86 C_{0}+1.283 C_{0}{ }^{2}-0.0078 C_{0}{ }^{3}-0.0006 C_{0}{ }^{4}
$$

Applying $C_{0}=-22.75$ we find $n=190$ tubes. The result is greater than the above of our method, which gave 163 tubes, and much greater than 150 tubes of the previous empirical equation in [10]. The deviation from our prediction is about $16.6 \%$ or 27 tubes more and from the empirical equation $26.7 \%$ or 40 tubes more, respectively.

(d) The empirical equation by Guo et al. [19] gives the shell inside diameter $D_{i}$. To compare it with our method, we apply the initial data of the heat exchanger: $d_{0}=19.05 \mathrm{~mm}$, $t=25.4 \mathrm{~mm}$. Using the same number of tubes, i.e. $n=151$, we obtain:

$$
\begin{gathered}
D_{i}=(1.1 \sqrt{n}-1) t+3 d_{0}= \\
=(1.1 \sqrt{151}-1) \times 25.4+3 \times 19.05=375.08 \mathrm{~mm}
\end{gathered}
$$

The calculated diameter is too larger compared with the value $D_{i}=336.55 \mathrm{~mm}$ of the heat exchanger, which means a deviation of about $11.4 \%$.

All the above comparisons showed that there are remarkable deviations from the exact solution when using available tables for tube counts or empirical equations.

\section{CONCLUSION}

The investigation of geometric characteristics of shell-andtube heat exchangers was successfully performed and the solution was presented explicitly in a simplified form for many tube arrangements and tube dimensions. A simple working equation for shell inside diameter was given and a characteristic parameter was introduced to calculate the geometric factors of the heat exchanger. The values of the characteristic parameter were calculated by a computer program and tabulated. The comparison of the results with those obtained through approximate equations available in the literature, showed noticeable deviations from the exact solution. The values of tube counts that were taken from tables of Handbooks also compared and appear to be somewhat lower than the exact solution of the present study. 


\section{APPENDIX}

Table A.1: Tube counts and parameter $f$ for pattern SQ-1

\begin{tabular}{|c|c|c|c|c|c|}
\hline $\begin{array}{l}\text { Tube } \\
\text { counts }\end{array}$ & Parameter & $\begin{array}{l}\text { Tube } \\
\text { counts }\end{array}$ & Parameter & $\begin{array}{l}\text { Tube } \\
\text { counts }\end{array}$ & Parameter \\
\hline$n$ & $f$ & $n$ & $f$ & $n$ & $f$ \\
\hline 1 & 0 & 277 & 18.43909 & 609 & 27.85678 \\
\hline 5 & 2 & 285 & 18.86796 & 613 & 28 \\
\hline 9 & 2.82843 & 293 & 18.97367 & 621 & 28.07134 \\
\hline 13 & 4 & 301 & 19.69772 & 633 & 28.28427 \\
\hline 21 & 4.47214 & 305 & 19.79899 & 641 & 28.42534 \\
\hline 25 & 5.65685 & 317 & 20 & 657 & 28.63564 \\
\hline 29 & 6 & 325 & 20.09975 & 665 & 28.84441 \\
\hline 37 & 6.32456 & 333 & 20.39608 & 673 & 29.12044 \\
\hline 45 & 7.21110 & 341 & 20.59126 & 681 & 29.52965 \\
\hline 49 & 8 & 349 & 20.88061 & 697 & 29.73214 \\
\hline 57 & 8.24621 & 357 & 21.26029 & 709 & 30 \\
\hline 61 & 8.48528 & 365 & 21.54066 & 717 & 30.06659 \\
\hline 69 & 8.94427 & 373 & 21.63331 & 725 & 30.26549 \\
\hline 81 & 10 & 377 & 22 & 733 & 30.46309 \\
\hline 89 & 10.19804 & 385 & 22.09072 & 741 & 30.52867 \\
\hline 97 & 10.77033 & 401 & 22.36068 & 749 & 30.59412 \\
\hline 101 & 11.31371 & 405 & 22.62742 & 757 & 31.04835 \\
\hline 109 & 11.66190 & 421 & 22.80351 & 761 & 31.11270 \\
\hline 113 & 12 & 429 & 23.32381 & 769 & 31.24100 \\
\hline 121 & 12.16552 & 437 & 23.40940 & 777 & 31.30495 \\
\hline 129 & 12.64911 & 441 & 24 & 793 & 31.62278 \\
\hline 137 & 12.80625 & 457 & 24.08319 & 797 & 32 \\
\hline 145 & 13.41641 & 465 & 24.16609 & 805 & 32.06244 \\
\hline 149 & 14 & 473 & 24.33105 & 821 & 32.24903 \\
\hline 161 & 14.14214 & 481 & 24.41311 & 829 & 32.31099 \\
\hline 169 & 14.42220 & 489 & 24.73863 & 845 & 32.55764 \\
\hline 177 & 14.56022 & 497 & 25.05993 & 853 & 32.80244 \\
\hline 185 & 15.23155 & 505 & 25.29822 & 861 & 32.98484 \\
\hline 193 & 15.62050 & 509 & 25.45584 & 869 & 33.10589 \\
\hline 197 & 16 & 517 & 25.61250 & 877 & 33.28663 \\
\hline 213 & 16.12452 & 529 & 26 & 885 & 33.52611 \\
\hline 221 & 16.49242 & 545 & 26.07681 & 889 & 33.94112 \\
\hline 225 & 16.97056 & 553 & 26.30589 & 901 & 34 \\
\hline 233 & 17.08801 & 561 & 26.68333 & 917 & 34.05877 \\
\hline 241 & 17.20465 & 569 & 26.83282 & 925 & 34.17601 \\
\hline 249 & 17.88854 & 577 & 26.90725 & 933 & 34.23449 \\
\hline 253 & 18 & 593 & 27.20294 & 941 & 34.40930 \\
\hline 261 & 18.11077 & 601 & 27.78489 & 949 & 34.52535 \\
\hline
\end{tabular}

\section{NOMENCLATURE}

$a=$ length of a circle chord parallel to $y$ axis (mm)

$A=$ integer number

$b=$ length of a circle chord parallel to $x$ axis ( $\mathrm{mm})$

$B=$ integer number

$B_{\mathrm{c}}=$ height of the baffle cut $(\mathrm{mm})$

$c=$ distance of a horizontal chord from $x$ axis (mm)

$C=$ clearance between shell and tube bundle $(\mathrm{mm})$

$C_{0}=$ parameter of empirical equation

$d_{0}=$ tube outer diameter $(\mathrm{mm})$

$D=$ circle diameter $(\mathrm{mm})$

$D_{b}=$ tube bundle diameter $(\mathrm{mm})$

$D_{i}=$ shell inside diameter $(\mathrm{mm})$

$f=$ characteristic parameter

$h=$ height of the equilateral triangle ( $\mathrm{mm}$ )

$i=$ number of holes at the circumference of a circle

$j=$ number of rows

$k=$ row numbering

$k_{1}=$ factor of empirical equation

$m, m_{1}, m_{2}=$ number of rows

$m_{1(y)}, m_{1(y 1)}, m_{2(y)}, m_{2(y 1)}=$ number of rows

$n=$ number of holes included in a circle
Table A.2: Tube counts and parameter $f$ for pattern SQ-2

Tube Parameter Tube Parameter Tube $\begin{gathered}\text { Tarameter } \\ \text { counts }\end{gathered}$

\begin{tabular}{crcccc}
$n n$ & $f$ & $n$ & $f$ & $n$ & $f$ \\
\hline 4 & 1.41421 & 360 & 21.21320 & 732 & 30.36445 \\
12 & 3.16228 & 368 & 21.40093 & 740 & 30.88689 \\
16 & 4.24264 & 376 & 21.58703 & 756 & 31.01612 \\
24 & 5.09902 & 384 & 21.95450 & 772 & 31.14482 \\
32 & 5.83095 & 392 & 22.13594 & 788 & 31.40064 \\
44 & 7.07107 & 400 & 22.67157 & 804 & 31.78050 \\
52 & 7.61577 & 408 & 22.84732 & 812 & 31.90611 \\
60 & 8.60233 & 424 & 23.02173 & 820 & 32.28003 \\
68 & 9.05539 & 432 & 23.19483 & 824 & 32.52691 \\
76 & 9.48683 & 440 & 23.53720 & 840 & 32.64965 \\
80 & 9.89950 & 448 & 23.70654 & 848 & 32.89377 \\
88 & 10.29563 & 460 & 24.04163 & 864 & 33.01515 \\
96 & 11.04536 & 468 & 24.20744 & 872 & 33.13608 \\
112 & 11.40175 & 484 & 24.69818 & 880 & 33.37664 \\
120 & 12.08305 & 492 & 25.01999 & 896 & 33.61547 \\
124 & 12.72792 & 500 & 25.17936 & 904 & 33.73426 \\
140 & 13.03840 & 524 & 25.49510 & 912 & 33.97058 \\
148 & 13.34166 & 532 & 25.80698 & 928 & 34.20526 \\
156 & 13.92839 & 540 & 25.96151 & 936 & 34.43835 \\
164 & 14.21267 & 548 & 26.41969 & 944 & 34.66987 \\
172 & 14.76482 & 556 & 26.57066 & 952 & 34.78505 \\
180 & 15.03330 & 560 & 26.87006 & 960 & 35.01428 \\
188 & 15.29706 & 576 & 27.01851 & 968 & 35.12834 \\
192 & 15.55635 & 584 & 27.16615 & 988 & 35.35534 \\
208 & 15.81139 & 592 & 27.31300 & 1004 & 35.46830 \\
216 & 16.55295 & 608 & 27.45906 & 1012 & 35.69314 \\
232 & 17.02939 & 616 & 27.89265 & 1020 & 35.80503 \\
240 & 17.26268 & 624 & 28.17801 & 1028 & 36.13862 \\
248 & 17.49286 & 632 & 28.31960 & 1036 & 36.24914 \\
256 & 17.72005 & 640 & 28.46050 & 1044 & 36.35932 \\
268 & 18.38478 & 648 & 28.60070 & 1052 & 36.68787 \\
276 & 18.60108 & 656 & 29.01724 & 1060 & 36.79674 \\
284 & 19.02630 & 680 & 29.15476 & 1076 & 37.01351 \\
300 & 19.23538 & 688 & 29.42788 & 1092 & 37.12142 \\
308 & 19.64688 & 692 & 29.69848 & 1108 & 37.33631 \\
316 & 19.84943 & 708 & 29.83287 & 1116 & 37.44329 \\
332 & 20.24846 & 716 & 29.96665 & 1124 & 37.65634 \\
348 & 21.02380 & 724 & 30.23243 & 1148 & 38.07887 \\
\hline & & & & &
\end{tabular}

$n_{1}=$ factor of empirical equation

$N_{c}=$ number of holes in the baffle window

$R=$ circle radius $(\mathrm{mm})$

$s=$ tube thickness $(\mathrm{mm})$

$t=$ tube pitch $(\mathrm{mm})$

$x, y, y_{1}=$ orthogonal axes

$x^{\prime}, y^{\prime}=$ inclined axes

$z_{k}, z_{0}, z_{1}=$ number of holes on a particular row

Greek Symbols

$\delta=$ clearance between adjacent tubes $(\mathrm{mm})$

Abbreviations

Int $=$ integer part of a number

SQ-1 = square tube pattern with a hole at the center

SQ-2 = square tube pattern without a hole at the center

TR-1 = triangular tube pattern with a hole at the center

TR-2 = triangular tube pattern without a hole at the center 
Table A.3: Tube counts and parameter $f$ for pattern TR-1

\begin{tabular}{|c|c|c|c|c|c|}
\hline $\begin{array}{c}\text { Tube } \\
\text { counts }\end{array}$ & Parameter & $\begin{array}{c}\text { Tube } \\
\text { counts }\end{array}$ & Parameter & $\begin{array}{c}\text { Tube } \\
\text { counts }\end{array}$ & Parameter \\
\hline$n$ & $f$ & $n$ & $f$ & $n$ & $f$ \\
\hline 1 & 0 & 385 & 20.78460 & 859 & 30.78960 \\
\hline 7 & 2 & 397 & 20.88062 & 871 & 31.04834 \\
\hline 13 & 3.46410 & 409 & 21.07130 & 877 & 31.17692 \\
\hline 19 & 4 & 421 & 21.16602 & 889 & 31.24100 \\
\hline 31 & 5.29150 & 433 & 21.63330 & 913 & 31.43246 \\
\hline 37 & 6 & 439 & 22 & 925 & 31.74902 \\
\hline 43 & 6.92820 & 451 & 22.27106 & 931 & 32 \\
\hline 55 & 7.21110 & 463 & 22.53886 & 955 & 32.18696 \\
\hline 61 & 8 & 475 & 22.71564 & 967 & 32.74142 \\
\hline 73 & 8.71780 & 499 & 23.06512 & 979 & 32.92416 \\
\hline 85 & 9.16516 & 511 & 23.57966 & 1003 & 33.04542 \\
\hline 91 & 10 & 517 & 24 & 1015 & 33.28664 \\
\hline 97 & 10.39230 & 535 & 24.24872 & 1027 & 33.40658 \\
\hline 109 & 10.58300 & 547 & 24.33104 & 1039 & 33.64520 \\
\hline 121 & 11.13552 & 559 & 24.57642 & 1045 & 34 \\
\hline 127 & 12 & 571 & 24.98000 & 1057 & 34.11744 \\
\hline 139 & 12.16552 & 583 & 25.05992 & 1069 & 34.17602 \\
\hline 151 & 12.49 & 595 & 25.53430 & 1075 & 34.64102 \\
\hline 163 & 13.11488 & 613 & 26 & 1099 & 34.69870 \\
\hline 169 & 13.85640 & 625 & 26.15340 & 1111 & 34.87120 \\
\hline 187 & 14 & 637 & 26.22976 & 1123 & 35.04284 \\
\hline 199 & 14.42220 & 649 & 26.45752 & 1135 & 35.15680 \\
\hline 211 & 15.09966 & 661 & 26.90724 & 1147 & 35.38362 \\
\hline 223 & 15.62050 & 673 & 27.05550 & 1159 & 35.55278 \\
\hline 235 & 15.87450 & 685 & 27.49546 & 1165 & 36 \\
\hline 241 & 16 & 691 & 27.71282 & 1177 & 36.05552 \\
\hline 253 & 16.37070 & 703 & 27.78488 & 1189 & 36.16628 \\
\hline 265 & 17.08800 & 721 & 28 & 1201 & 36.38682 \\
\hline 271 & 17.32050 & 733 & 28.21348 & 1213 & 36.49658 \\
\hline 283 & 17.43560 & 745 & 28.35490 & 1225 & 36.66060 \\
\hline 295 & 17.77638 & 757 & 28.84442 & 1237 & 36.71512 \\
\hline 301 & 18 & 769 & 29.05168 & 1261 & 37.04052 \\
\hline 313 & 18.33030 & 793 & 29.46184 & 1273 & 37.36308 \\
\hline 337 & 19.07878 & 805 & 29.59730 & 1285 & 37.46998 \\
\hline 349 & 19.28730 & 817 & 29.86636 & 1303 & 38 \\
\hline 361 & 19.69772 & 823 & 30 & 1309 & 38.10512 \\
\hline 367 & 20 & 835 & 30.19934 & 1333 & 38.15756 \\
\hline 379 & 20.29778 & 847 & 30.26550 & 1345 & 38.31448 \\
\hline
\end{tabular}

Table A.4: Tube counts and parameter $f$ for pattern TR-2

\begin{tabular}{|c|c|c|c|c|c|}
\hline $\begin{array}{l}\text { Tube } \\
\text { counts }\end{array}$ & Parameter & $\begin{array}{l}\text { Tube } \\
\text { counts }\end{array}$ & Parameter & $\begin{array}{l}\text { Tube } \\
\text { counts }\end{array}$ & Parameter \\
\hline$n$ & $f$ & $n$ & $f$ & $n$ & $f$ \\
\hline 3 & 1.15470 & 231 & 16.04161 & 498 & 23.35237 \\
\hline 6 & 2.30940 & 240 & 16.16581 & 504 & 23.43786 \\
\hline 12 & 3.05505 & 246 & 16.28906 & 510 & 23.69247 \\
\hline 18 & 4.16333 & 252 & 16.65333 & 522 & 23.86071 \\
\hline 21 & 4.61880 & 258 & 16.77299 & 528 & 24.02776 \\
\hline 27 & 5.03322 & 270 & 17.00980 & 534 & 24.11086 \\
\hline 30 & 5.77350 & 276 & 17.24336 & 540 & 24.19366 \\
\hline 36 & 6.11010 & 282 & 17.47379 & 546 & 24.44040 \\
\hline 42 & 6.42910 & 288 & 17.92577 & 552 & 24.68468 \\
\hline 48 & 7.02377 & 294 & 18.03700 & 558 & 24.84620 \\
\hline 54 & 7.57188 & 306 & 18.14754 & 570 & 25.00667 \\
\hline 63 & 8.08290 & 309 & 18.47521 & 576 & 25.16611 \\
\hline 69 & 8.32666 & 321 & 18.58315 & 588 & 25.32456 \\
\hline 75 & 9.01850 & 327 & 18.90326 & 591 & 25.40341 \\
\hline 78 & 9.23760 & 333 & 19.00877 & 597 & 25.48202 \\
\hline 84 & 9.45163 & 339 & 19.21805 & 603 & 25.71640 \\
\hline 90 & 9.86577 & 345 & 19.42507 & 609 & 25.79406 \\
\hline 96 & 10.06645 & 348 & 19.62991 & 615 & 26.02563 \\
\hline 102 & 10.26320 & 354 & 19.73153 & 627 & 26.10236 \\
\hline 114 & 11.01514 & 366 & 20.03331 & 633 & 26.40707 \\
\hline 120 & 11.37248 & 372 & 20.13289 & 636 & 26.55811 \\
\hline 123 & 11.54701 & 378 & 20.23199 & 648 & 26.63331 \\
\hline 129 & 11.71893 & 384 & 20.42874 & 654 & 26.85765 \\
\hline 135 & 12.05543 & 390 & 20.52641 & 660 & 27.00617 \\
\hline 141 & 12.22020 & 396 & 20.81666 & 672 & 27.15388 \\
\hline 144 & 12.70171 & 402 & 21.00794 & 678 & 27.22744 \\
\hline 150 & 12.85820 & 408 & 21.19748 & 690 & 27.30079 \\
\hline 156 & 13.01281 & 420 & 21.38535 & 696 & 27.59227 \\
\hline 168 & 13.31666 & 426 & 21.57159 & 702 & 27.73686 \\
\hline 174 & 13.61372 & 435 & 21.93931 & 714 & 28.02380 \\
\hline 180 & 14.04754 & 447 & 22.03028 & 720 & 28.09508 \\
\hline 186 & 14.18920 & 453 & 22.12088 & 726 & 28.30783 \\
\hline 192 & 14.46836 & 459 & 22.30097 & 732 & 28.37840 \\
\hline 198 & 14.74223 & 465 & 22.47962 & 738 & 28.44879 \\
\hline 207 & 15.01111 & 471 & 22.74496 & 744 & 28.58904 \\
\hline 213 & 15.14376 & 477 & 23.00725 & 750 & 28.72862 \\
\hline 219 & 15.27525 & 480 & 23.09401 & 753 & 28.86751 \\
\hline 225 & 15.53491 & 492 & 23.18045 & 759 & 28.93671 \\
\hline
\end{tabular}

\section{REFERENCES}

[1] D. Q. Kern, Process Heat Transfer. McGraw-Hill, New York, 1950.

[2] K. J. Bell, Final report of the cooperative research program on shell and tube heat exchangers. University of Delaware, Engineering Experimental Station, Bulletin No. 5, Newark, Delaware, 1963.

[3] M. Serna, and A. Jiménez, A compact formulation of the Bell -Delaware method for heat exchanger design and optimization. Chem. Eng. Res. Des., Vol. 83 (A5): pp. 539-550, 2005.

[4] J. Taborek, Shell-and-Tube Heat Exchangers. In: Schlünder, E.U. (ed), Heat Exchanger Design Handbook, Section 3.3. Hemisphere Publishing Corp., USA, 1983.

[5] J. R. Thome, Wolverine Engineering Data Book III. Wolverine Tube Inc., USA, 2006.

[6] S. Kakac, and H. Liu, Heat Exchangers, $2^{\text {nd }}$ ed. CRC Press, New York, 2002.

[7] R. K. Shah, and D. P. Sekulic, Fundamentals of Heat Exchanger Design. John Wiley \& Sons, Inc., Hoboken, New Jersey, 2003.

[8] R. W. Serth, and T. G. Lestina, Process Heat Transfer, $2^{\text {nd }}$ ed. Elsevier Inc., USA, 2014.

[9] D. W. Green, and R. H. Perry, Perry's Chemical Engineers' Handbook, $8^{\text {th }}$ ed. McGraw-Hill, Inc., USA, 2008.

[10] R. K. Sinnott, Chemical Engineering Design. Coulson \& Richardson's, Chemical Engineering series, Vol. 6, $4^{\text {th }}$ ed. Elsevier Butterworth -Heinemann, 2005.

[11] P. S. Phadke, "Determining tube counts for shell and tube exchangers". Chem. Eng. J., September 3, Vol. 91, pp. 65-68, 1984.
[12] K. Muralikrishna, and U. V. Shenoy, "Heat exchanger design targets for minimum area and cost". Trans. IChemE, Part A, Chem. Eng. Res. Des., Vol 78, No. 2, pp. 161-167, 2000.

[13] M. Serna-Gonzalez, J. M. Ponce-Ortega, A. J. Castro-Montoya, and A. Jimenez-Gutierrez, "Feasible design space for shell-and-tube heat exchangers using the Bell-Delaware method". Ind. Eng. Chem. Res., Vol. 46, No. 1, pp. 143-155, 2007.

[14] A. S. Sahin, B. Kilic, and U. Kilic, "Design and economic optimization of shell and tube heat exchangers using Artificial Bee Colony (ABC) algorithm”. Energy Convers. Manage., Vol. 52, pp. 3356-3362, 2011.

[15] S. Fettaka, J. Thibault, and Y. Gupta, "Design of shell-and-tube heat exchangers using multiobjective optimization". Int. J. Heat Mass Transfer, Vol. 60, pp. 343-354, 2013.

[16] TEMA, Standards of the Tubular Exchange Manufacturers Association. J. Harrison (ed.), New York, 1999.

[17] F. Tan, and S. Fok, "An educational tool for heat exchanger design". Comput. Appl. Eng. Educ., Vol. 14, No. 2, pp. 77-89, 2006.

[18] S. T. M Than, K. A. Lin, and M. S. Mon, "Heat Exchanger Design". Int. J. of Mechanical and Mechatronics Engineering, Vol. 2, No. 10, 2008.

[19] J. Guo, L. Cheng, and M. Xu, "Optimization design of shell-and-tube heat exchanger by entropy generation minimization and genetic algorithm". Appl. Therm. Eng., Vol. 29, pp. 2954-2960, 2009. 